\title{
Evidence based community mobilization for dengue prevention in Nicaragua and Mexico (Camino Verde, the Green Way): cluster randomized controlled trial
}

\author{
Neil Andersson,1,2 Elizabeth Nava-Aguilera, ${ }^{1}$ Jorge Arosteguí, ${ }^{3}$ Arcadio Morales-Perez, ${ }^{1}$ \\ Harold Suazo-Laguna, ${ }^{3}$ José Legorreta-Soberanis, ${ }^{1}$ Carlos Hernandez-Alvarez, ${ }^{3}$ \\ Ildefonso Fernandez-Salas, ${ }^{4}$ Sergio Paredes-Solís, ${ }^{1}$ Angel Balmaseda, ${ }^{5}$ Antonio Juan Cortés-Guzmán, ${ }^{6}$ \\ René Serrano de los Santos, ${ }^{1}$ Josefina Coloma, ${ }^{7}$ Robert J Ledogar, ${ }^{8}$ Eva Harris ${ }^{7}$
}

\begin{abstract}
For numbered affiliations see end of article.

Correspondence to:

N Andersson

andersson@ciet.org

Additional material is published online only. To view please visit the journal online (http://dx.doi. org/10.1136/bmj.h3267)

Cite this as: BMJ 2015;351:h3267 doi: $10.1136 /$ bmj.h3267

Accepted: 11 June 2015
\end{abstract}

\section{ABSTRACT}

\section{OBJECTIVE}

To test whether community mobilization adds effectiveness to conventional dengue control.

DESIGN

Pragmatic open label parallel group cluster randomized controlled trial. Those assessing the outcomes and analyzing the data were blinded to group assignment. Centralized computerized randomization after the baseline study allocated half the sites to intervention, stratified by country, evidence of recent dengue virus infection in children aged 3-9, and vector indices.

SETTING

Random sample of communities in Managua, capital of Nicaragua, and three coastal regions in Guerrero State in the south of Mexico.

\section{PARTICIPANTS}

Residents in a random sample of census enumeration areas across both countries: 75 intervention and 75 control clusters (about 140 households each) were randomized and analyzed (60 clusters in Nicaragua and 90 in Mexico), including 85182 residents in 18838 households.

INTERVENTIONS

A community mobilization protocol began with community discussion of baseline results. Each

\section{WHAT IS ALREADY KNOWN ON THIS TOPIC}

Current dengue control rests heavily on using the organophosphate pesticide temephos (Abate) in household water storage containers

The dengue pandemic has continued to grow despite widespread use of temephos, and resistance to this pesticide is well documented. Space spraying with other pesticides is common but of little proved value

Though several studies have shown an impact of community interventions on vector control, none has shown an impact on dengue illness or serological evidence of infection

\section{WHAT THIS STUDY ADDS}

The Camino Verde (Green Way) is pesticide-free evidence based community mobilization, each community choosing and implementing its own mix of dengue prevention actions based on local vector reservoirs and community resources The project had a positive impact on serological evidence of dengue virus infection in children, reported illness at all ages, and all dengue vector control indices This is the first report of serological evidence of impact of community interventions Serological evidence could not confirm any protective effect of temephos against infection with dengue virus-overall or in any subgroups intervention cluster adapted the basic interventionchemical-free prevention of mosquito reproductionto its own circumstances. All clusters continued the government run dengue control program.

\section{MAIN OUTCOME MEASURES}

Primary outcomes per protocol were self reported cases of dengue, serological evidence of recent dengue virus infection, and conventional entomological indices (house index: households with larvae or pupae/households examined; container index: containers with larvae or pupae/containers examined; Breteau index: containers with larvae or pupae/households examined; and pupae per person: pupae found/number of residents). Per protocol secondary analysis examined the effect of Camino Verde in the context of temephos use.

RESULTS

With cluster as the unit of analysis, serological evidence from intervention sites showed a lower risk of infection with dengue virus in children (relative risk reduction $29.5 \%, 95 \%$ confidence interval $3.8 \%$ to $55.3 \%$ ), fewer reports of dengue illness (24.7\%, $1.8 \%$ to $51.2 \%$ ), fewer houses with larvae or pupae among houses visited (house index) (44.1\%, $13.6 \%$ to $74.7 \%)$, fewer containers with larvae or pupae among containers examined (container index) (36.7\%, 24.5\% to 44.8\%), fewer containers with larvae or pupae among houses visited (Breteau index) (35.1\%, 16.7\% to 55.5\%), and fewer pupae per person $(51.7 \%, 36.2 \%$ to $76.1 \%)$. The numbers needed to treat were 30 (95\% confidence interval 20 to 59) for a lower risk of infection in children, 71 (48 to 143) for fewer reports of dengue illness, 17 (14 to 20) for the house index, 37 ( 35 to 67) for the container index, 10 ( 6 to 29) for the Breteau index, and 12 (7 to 31) for fewer pupae per person. Secondary per protocol analysis showed no serological evidence of a protective effect of temephos.

CONCLUSIONS

Evidence based community mobilization can add effectiveness to dengue vector control. Each site implementing the intervention in its own way has the advantage of local customization and strong community engagement.

TRIAL REGISTRATION

ISRCTN27581154

\section{Introduction}

Dengue is a major international health problem, with some 100 million cases and 400 million infections a year globally. ${ }^{12}$ In most countries, contemporary dengue 
control depends on placing an organophosphate larvacide-temephos (Abate)-in water storage containers that are potential breeding sites for the main vector Aedes aegypti. ${ }^{3}$ This has not curbed dengue epidemics. Space spraying with other pesticides is common, although of little proved value in dengue control. ${ }^{4}$ Multiple serotypes of dengue virus continue to spread worldwide, while A aegypti resistance to temephos increases. . $^{5-8}$

Failure of vertically managed pesticide distribution has increased interest in primary healthcare approaches, with community engagement to reduce mosquito breeding sites. A 2007 systematic review reported weak evidence that this might control dengue; ${ }^{9}$ none of the studies reviewed used clustered designs, however, despite recognition that mosquitoes fly between households and community mobilization is a group phenomenon. Subsequently, cluster randomized trials in Cuba and India showed impact of community mobilization on vector indices, ${ }^{1011}$ as did community volunteers in Thailand. ${ }^{12} \mathrm{~A}$ 2011 review of 22 studies of education for community dengue control confirmed effectiveness in reducing entomological indices, though no study measured infection with dengue virus. ${ }^{13}$ A recent review of 14 studies of Bacillus thuringiensis israelensis reported evidence of impact on the number of Aedes but again little evidence of impact on risk of dengue. ${ }^{14}$ The literature suggests that non-pesticide measures should prevent dengue, but there is little direct evidence to confirm health benefits.

A four year feasibility study in Managua developed a non-randomized pesticide-free intervention (10 intervention and 20 control clusters, 132 houses/clusters, and 3300 children aged 3-9). Community volunteers used serological and entomological evidence to engage residents in interventions: household visits, demonstration of mosquito eggs and larvae/pupae, and simple tools for elimination of breeding sites. All 5596 households allowed examination of vector reservoirs around the home; all but 21 answered questions about recent illness. Intervention communities showed a lower risk of dengue. ${ }^{15}$ Nicaragua's history of community mobilization, however, raised issues of relevance to other settings.

This cluster randomized controlled trial assessed the added value of evidence based community engagement in dengue prevention-in Managua by scaling up already tested strategies, in southern Mexico by implementing these strategies in environments less hospitable to them, and in both places in a random sample of census enumeration areas (fig 1).

\section{Methods}

The hypothesis was that informed community mobilization adds effectiveness to government run dengue control programs in Managua, Nicaragua, and the coastal regions of Mexico's Guerrero state. ${ }^{16}$ Specific objectives were to determine cluster level entomological and serological status; translate these results into action against dengue through dialogue with participating communities; and identify cluster level real life impact of informed community mobilization.

After a baseline study in a random sample of census enumeration areas in Nicaragua and Mexico, we randomly allocated one half to receive the intervention. These clusters followed a protocol to engage communities in dengue prevention alongside the usual government dengue prevention activities for one year. We measured the impact during a second study at the end of the following dengue season.

\section{Patient involvement}

Patients who had previously had dengue and their families were not involved in setting the research question or the outcome measures, but they were they intimately involved in design and implementation of the intervention. Patients and their families were also central to dissemination of the baseline information, which helped to motivate community involvement during and beyond the study.

\section{Participants}

Participants were all residents in a stratified last stage random sample of enumeration areas ${ }^{17}$ (the geographic area canvassed by one census representative) from the latest censuses, with 60 clusters in Nicaragua and 90 in Mexico. We included all children aged 3-9 whose parents consented to them providing a saliva sample. The Guerrero sample covered three coastal regions (Costa Grande, Acapulco, and Costa Chica), stratified for population size and dengue prevention programs. The Nicaraguan sample came from the capital city of Managua, where a quarter of the national population lives. This sampling frame excluded 17 wealthy enumeration areas, where residents typically make private security arrangements and do not participate in public health initiatives for the popular neighborhoods. Table A in appendix 1 shows baseline demographic, infrastructural, and socioeconomic factors that could influence dengue transmission.

Before the baseline surveys, we received permission from the mayoral office and community leaders. Individual consent was also read to every respondent, all of whom were told that they were free to decline to answer any question they opted not to answer. Parents gave individual consent for each child who contributed saliva samples. The interventions started with requesting permission from community leaders.

\section{Interventions}

Before randomization, interviewers returned entomology results to all households and individual serology results to parents of the children who had provided saliva samples for serological testing. All clusters continued the official government dengue control programs (monthly deposits of temephos sachets in water storage of all households and space spraying), and all participated in a baseline and follow-up trial survey.

Interventions covered self identified neighborhoods and were not confined to the 140 household impact assessment clusters within them. All intervention communities followed the same intervention protocol, initiating the community engagement through three protocol steps:

- The researchers asked permission from community leaders and engaged them in discussion of baseline evidence 

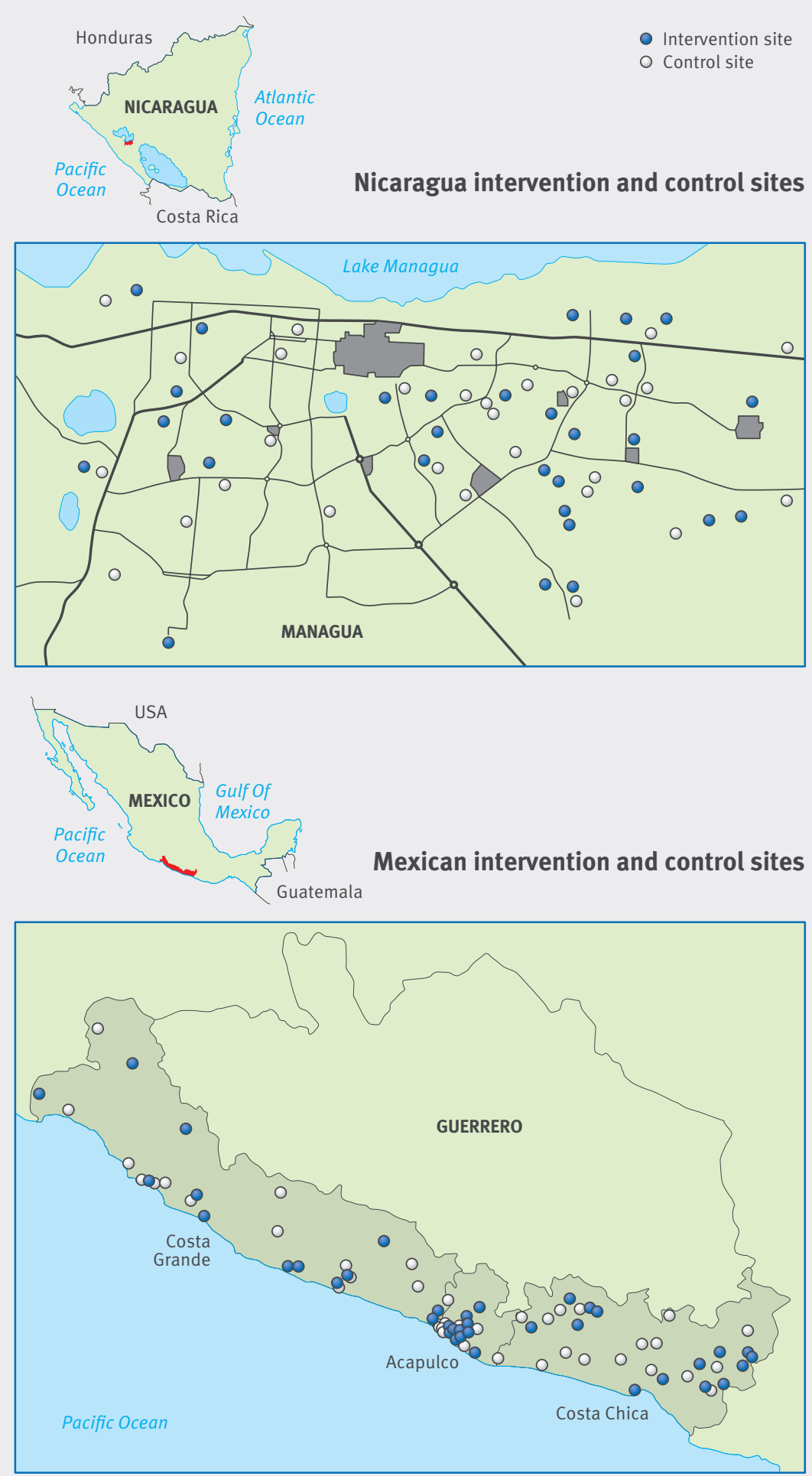

Fig 1 Areas covered by study of evidence based community mobilization for dengue prevention in Nicaragua and Mexico figures like school teachers, and some identified by door to door invitation. In Managua, facilitators were former volunteers from the feasibility study; in Mexico, they were recent university graduates in social sciences. Communities opted for a range of activities to share basic information on the mosquito life cycle and how to interrupt it (emptying, brushing/scrubbing the interior walls of, or covering receptacles hosting mosquito eggs or larvae); community events to raise awareness, like puppet shows and basketball tournaments; clean up campaigns focused on unoccupied and public premises; introduction of fish into water storage containers (Mexico only); and other activities listed in appendix 2

- Through local community leaders in Nicaragua and directly in Mexico, the research team invited volunteers (brigadistas) from participating communities to receive training as organizers and educators. In all intervention communities, brigadistas visited households and schools to show evidence of larval/pupal infestation in water receptacles, to inform households and schools of the mosquito's life cycle, and to counsel on ways to interrupt the cycle. Brigadistas also added interventions as their community work advanced (see appendix 2). In Nicaragua, brigadistas received no remuneration; in Mexico, they received allowances for travel, lunch, and child care on the days they worked.

Exchanges between countries informed by the Nicaraguan feasibility study sought commonality of function by assuring the same protocol to generate community led interventions rather than uniformity of the specific actions. ${ }^{18}$ Mid-stream peer evaluation assessed fidelity to the intervention and dynamics of engagement and encouraged interaction among neighborhoods.

The baseline survey included an entomological survey in the dry season, paired saliva samples before and after the dengue epidemic, and one questionnaire related to dengue and one related to social capital/ costs. The baseline survey took place between August 2010 and January 2011. The follow-up impact assessment likewise included an entomological survey in the wet and dry season, paired saliva samples before and after the dengue season, and one questionnaire related to dengue and one related to social capital/costs. In Nicaragua, this was from August 2012 to January 2013 and in Mexico from August to November 2012.

Sequence of steps-The baseline surveys and results from the paired saliva samples preceded, and provided stratifying data for, the randomization. Randomization was followed by the intervention, followed by the impact assessment. The baseline and impact assessments were each made up of two surveys to allow for collection of the paired saliva samples. Serological status from saliva samples was based on differences between the first and second sample. This also enabled vector assessments in the wet and dry season; we report only the dry season entomology assessment.

Timelines-Figure 2 summarizes the timings of the study. 


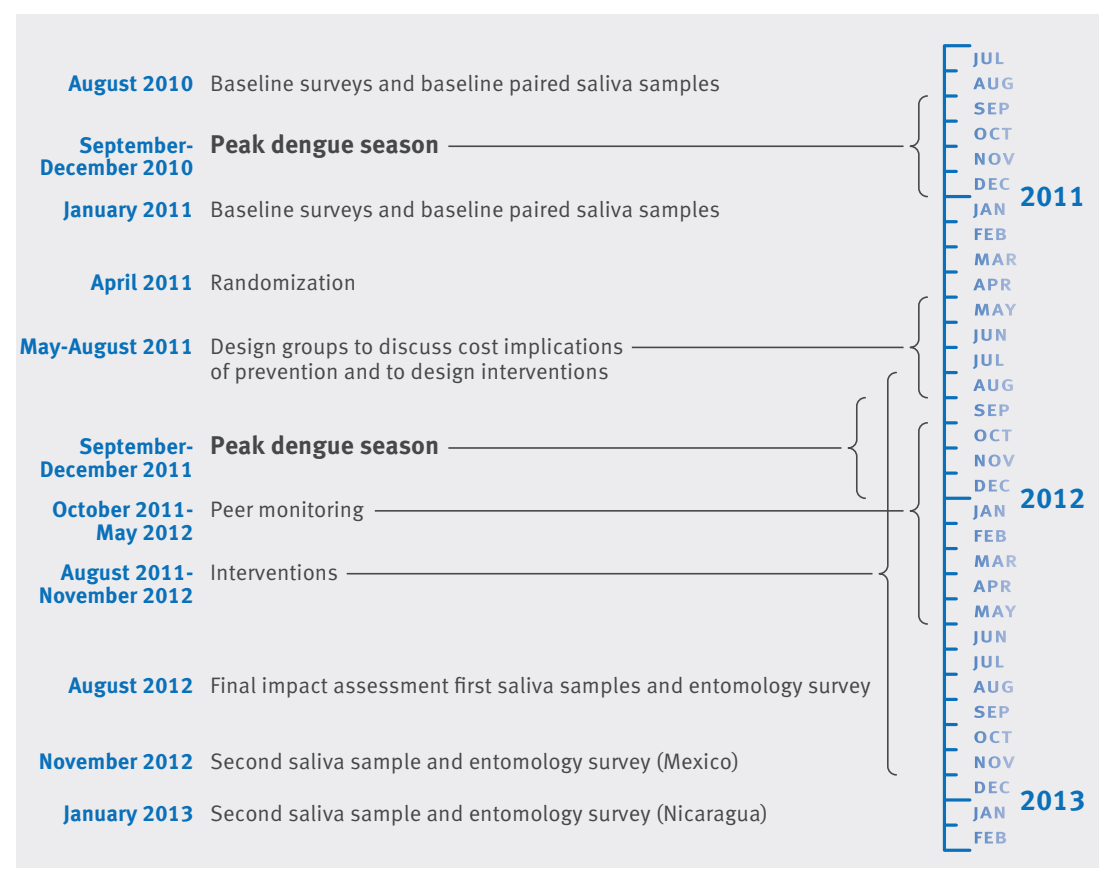

Fig 2 | Timeline of study of evidence based community mobilization for dengue prevention in Nicaragua and Mexico and it is harder to detect incident secondary infections with our assay. The assay relied on non-invasive saliva samples to estimate incidence of infection in large numbers of participants (low refusal rate). We used self reported dengue as an outcome indicator because, notwithstanding its imperfect reliability, this does include all age groups. The funds and scope of the trial did not permit active surveillance to capture all cases of dengue confirmed by laboratory.

Dengue illness-Self reported cases of dengue were recorded from responses to a direct question about each household member in turn "Did this person suffer from dengue in the last year?”

Vector assessment-Entomology evaluators inspected indoor and outdoor containers, tires, flower vases, water barrels, wash basins, tarpaulins, and discarded containers. The impact assessment focused on household breeding sites; interventions also dealt with breeding sites in open lots and public spaces. Fieldworkers who were not part of the intervention used nets to collect all larvae and pupae from each container. Supervisors transported samples in labeled plastic bags to the respective entomology laboratories for identification. Entomological indices included A aegypti house index (households with larvae or pupae/households examined), container index (containers with larvae or pupae/containers examined), Breteau index (containers with larvae or pupae/households examined), and pupae per person (pupae found/number of residents). There was onebaseline collection in the dry season, and a final assessment in the wet and the dry season. We report here the final assessment in the dry season.

Per protocol secondary outcomes focused on information from face-to-face interviews regarding conscious knowledge about dengue and its prevention and control; attitudes (respondents who agreed to a direct question that temephos and fumigation are the best way to avoid mosquitoes/households interviewed); subjective norms (what neighbors do) and positive deviations from a negative norm; intention to change behavior in the future and to implement preventive action; agency (collective and individual self efficacy)-respondents who said communities can themselves control dengue/households interviewed; discussion-talk with neighbors about how to avoid mosquitoes or prevent dengue; action (interventions, practices)-households that purchased pesticides in the past month; and health literacy, resilience and social capital (see table 3). A separate study, now in progress, will report on the economic costs and implications for sustainability.

\section{Randomization}

Sequence generation-Each cluster was a census enumeration area. Computer generated random numbers allocated interventions in each stratum; strata comprised all eligible clusters in each country divided into region and, from the baseline survey, categories of risk of dengue virus infection in children aged 3-9 and vector indices.

Allocation concealment-Intervention status was disclosed once interventions began in both countries.

Implementation-A sampling statistician not engaged in the study conducted random selection from the population census and central random allocation of clusters to intervention. 


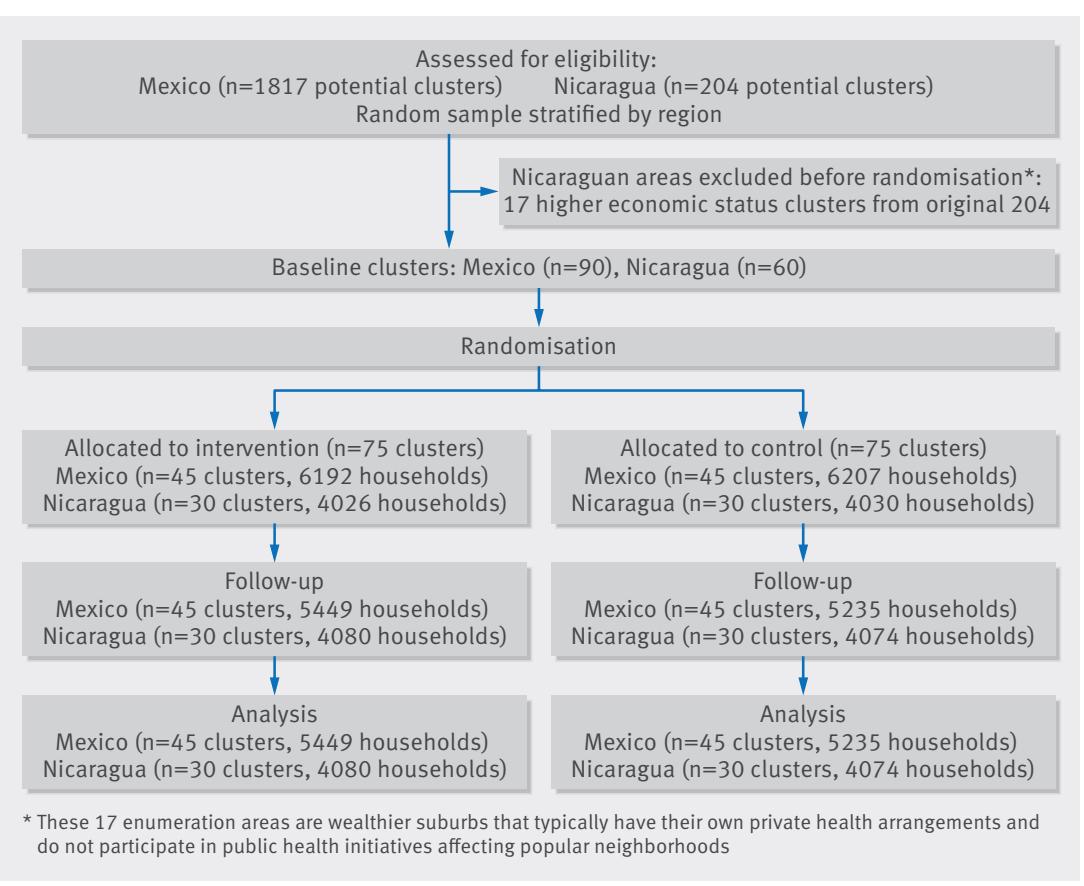

Fig 3 Identification and flow of clusters and households in study of community mobilization in Nicaragua and Mexico for dengue prevention

Masking-Intervention efforts were obvious to residents in intervention sites, and some outcomes (like collective self efficacy) could be influenced by this knowledge. The main outcomes (serological evidence of recent risk of infection and entomological indices) are less susceptible to this bias. As far as possible, interviewers and entomological assessors in the follow-up survey were unaware of intervention status. Data operators were unaware of intervention status.

\section{Estimates of sample size}

The 2004-08 Managua feasibility study facilitated estimation of required sample size. Control communities showed serological evidence of recent dengue virus infection in children: $1.5 \%$ to $7.4 \%$ across 20 non-intervention clusters (intraclass correlation $=0.18$, estimated by dividing variance between clusters by total variance in the control group). Serology of 40 children in each of 150 clusters (75 clusters per arm) would detect a 33.3\% reduction in risk of infection (4.5\% to 3\%) with $80 \%$ power at a significance level of $5 \%$. These calculations assumed equal cluster size, homogeneous treatment effect, and a two sided test with cluster as unit of primary analysis in unmatched parallel groups. Calculations used the approach of Taylor and Bosch for parallel group cluster trials. ${ }^{22}$

\section{Statistical methods}

Data entry and security-Data operators ignorant of intervention status entered household responses twice, with verification of discordant entries from the original questionnaires. A data manager checked digitized data for logical errors.

Principal analysis-We used a $t$ test in an intention to treat analysis of cluster specific rates. ${ }^{23}$ We included in the analyses the two Nicaraguan intervention sites that declined to participate. Continuous variables in each site were proportions of children showing a more than twofold increase in IgG units between two samples from the same child before and after the dengue season, households with a reported dengue case, and thresholds of conventional entomological indices (above 0 ). We also used cluster as the unit analysis for our secondary outcomes-household responses to an administered questionnaire. We estimated relative risk reduction (RRR) as 1-relative risk, using variance of the relative risk (Delta method) to estimate confidence intervals. We estimated the number needed to treat (NNT) as the inverse of risk difference (RD) and intraclass correlation (ICC) by dividing the variance between clusters by the variance between and within clusters across the control series. ${ }^{24}$

Secondary analysis-Per protocol bivariate and then multivariate analysis evaluated impact (evidence of recent dengue virus infection in the household) of living in intervention sites in the context of other factors that might influence infection, mostly household responses to an administered questionnaire. These included temephos exposure (sachets found in water storage containers), household pesticide purchase, household water supply ("How often does your household receive water?”), baseline level of dengue virus infection, and sex of the respondent. We included all those significant at the $5 \%$ level (adjusted for cluster) in a generalized linear mixed model (GLMM) with country and cluster as random effects. We report the odds ratio and adjusted odds ratio. Planned subgroup analysis focused on geographic variations of the protective effect, especially population density (urban/rural) and the particular choices of intervention.

Missing data-The follow-up survey included new arrivals in both groups but did not follow those leaving. People who decline to respond might be less involved with dengue control, thus reducing the measured effect. We used Amelia $\mathrm{II}^{25}$ to impute values for missing data with an expectation-maximization algorithm for the primary outcome household report of dengue illness. Estimates reconciled data from 10 imputed datasets with Rubin's approach ${ }^{26}$ in the R package Zelig ${ }^{27}$.

Software-CIETmap ${ }^{28}$ is open source software with a Windows-like interface for the open source statistical programing language $R$.

\section{Results}

\section{Participant flow}

Figure 3 describes the numbers of clusters and participants completing the study protocol and analyzed for primary outcomes. There were no deviations from protocol.

\section{Baseline data}

Table 1 lists 2010-11 baseline results for individuals and clusters in intervention and control groups, showing similar risks of dengue virus infection in households with children aged 3-9 (around 9\% in Mexico and twice that rate in Nicaragua), household reports of dengue in the past year (6\% in Mexico and 9\% in Nicaragua), and proportion of households positive for A aegypti larvae/ pupae (16\% in Mexico and 20\% in Nicaragua). 
Table 1| Group and individual baseline data at random assignment to intervention and control in study of community mobilization (intervention) in Nicaragua and Mexico for dengue prevention. Figures are numbers (percentage)

\begin{tabular}{|c|c|c|c|c|}
\hline & \multicolumn{2}{|l|}{ Mexico } & \multicolumn{2}{|l|}{ Nicaragua } \\
\hline & Intervention & Control & Intervention & Control \\
\hline \multicolumn{5}{|l|}{ Individual and household rates at baseline } \\
\hline $\begin{array}{l}\text { Serology: households with evidence of recent dengue virus infection in children aged 3-9/ } \\
\text { No of households contributing paired samples (\%) }\end{array}$ & $317 / 3331(9.5)$ & 259/3051 (8.5) & $316 / 1747(18)$ & $300 / 1765(17)$ \\
\hline Self reported dengue illness: households with dengue/households surveyed (\%) & $400 / 6191(6.5)$ & $389 / 6207(6.3)$ & $330 / 4023(8.2)$ & $349 / 4024(8.7)$ \\
\hline House index: No of households with larvae or pupae/households surveyed (\%) & $1020 / 6192(16.5)$ & $1014 / 6207(16.3)$ & $798 / 4026(20)$ & $750 / 4030(19)$ \\
\hline $\begin{array}{l}\text { Collective self efficacy: respondents who believe community can prevent dengue on its } \\
\text { own/households surveyed (\%) }\end{array}$ & $4716 / 6103(77.3)$ & $4650 / 6126(75.9)$ & 1991/3940 (51) & $2131 / 3967(54)$ \\
\hline $\begin{array}{l}\text { Purchased pesticide: households that purchased pesticide in past month/households } \\
\text { surveyed (\%) }\end{array}$ & $2631 / 6155(42.7)$ & $2767 / 6170(44.8)$ & 2092/3931 (53) & $2095 / 3955(53)$ \\
\hline \multicolumn{5}{|l|}{ Cluster factors at baseline* } \\
\hline No of clusters & 45 & 45 & 30 & 30 \\
\hline Risk of recent dengue virus infection: No of clusters with higher than country rate at baseline & $15(33)$ & $17(38)$ & $13(43)$ & $14(47)$ \\
\hline $\begin{array}{l}\text { Self reported dengue cases in past year: No of clusters with higher than country rate at } \\
\text { baseline }\end{array}$ & $18(40)$ & $19(42)$ & $14(47)$ & $13(43)$ \\
\hline House index: No of clusters with higher than country rate at baseline & $18(40)$ & $22(49)$ & $12(40)$ & $10(33)$ \\
\hline Collective self efficacy: No of clusters with higher than country rate at baseline & $23(51)$ & $26(58)$ & $12(40)$ & $15(50)$ \\
\hline Purchased pesticide: No of clusters with higher than country rate at baseline & $21(47)$ & $22(49)$ & $14(47)$ & $16(53)$ \\
\hline
\end{tabular}

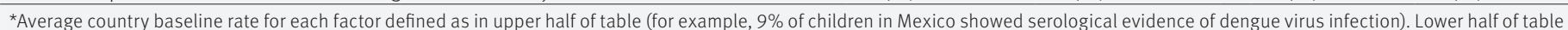
is proportion of clusters in intervention and control groups where average was higher than respective country baseline rate.

\section{Numbers analyzed}

The study included 85182 residents in 18838 households in 150 clusters (1414 (7\%) declined). Some 9499 children in 6698 households contributed saliva samples for the impact estimation (37 (0.4\%) declined), and evaluators examined 70388 vector breeding sites (table 2).

\section{Outcomes and estimation}

Table 3 and appendix 3 show primary outcomes and confidence intervals for each group with cluster as the unit of analysis. Intervention clusters had significantly lower rates of all primary outcomes- risk of dengue virus infection in children aged 3-9 old (relative risk reduction $29.5 \%$, 95\% confidence interval 3.8\% to $55.3 \%$; number needed to treat 30, 95\% confidence interval 20 to 59). Applied to our study population, this implies 33 young children per 1000 were spared increased levels of IgG across paired samples (absolute rate $11.3 \%$ in intervention children and $14.6 \%$ in control children, table 3$)$. Intervention clusters also reported fewer dengue cases in the past year $(24.7 \%, 1.8 \%$ to $51.2 \%$; number needed to treat 71,48 to 143 ). Applied to our study population, this implies 14 households per 1000 were spared having a case of dengue (absolute rates of $5.7 \%$ in intervention households and $7.1 \%$ in control households).

There were also significant reductions in A aegypti larvae and pupae in the intervention group, again with cluster as the unit of analysis. The relative risk reductions were $44.1 \%$ (95\% confidence interval $13.6 \%$ to $74.7 \%$ ) for a lower house index, $36.7 \%$ (24.5\% to $44.8 \%$ ) for the container index, $35.1 \%$ (16.7\% to $55.5 \%)$ for the Breteau index, and $51.7 \%$ (36.2\% to $76.1 \%$ ) for fewer pupae per person. The numbers needed to treat were 17 (14 to 20) for the house index, 37 (35 to 67) for the container index, 10 (6 to 29) for the Breteau index, and 12 (7 to 31) for fewer pupae per person.

Table 3 shows these parameters in a cluster analysis, the effect size (absolute difference and relative risk reduction estimated as 1-relative risk), precision

Table 2 | Numbers of individuals and households available for follow-up in study of community mobilization (intervention) in Nicaragua and Mexico for dengue prevention

\begin{tabular}{|c|c|c|c|c|c|}
\hline & \multicolumn{2}{|l|}{ Mexico } & \multicolumn{2}{|l|}{ Nicaragua } & \multirow[b]{2}{*}{ Total } \\
\hline & Intervention & Control & Intervention & Control & \\
\hline Clusters & 45 & 45 & 30 & 30 & 150 \\
\hline Households interviewed & 5449 & 5235 & 4080 & 4074 & 18838 \\
\hline Mean (range) No of households per cluster & $121(72-155)$ & $116(61-163)$ & $136(126-152)$ & $136(132-140)$ & $126(61-163)$ \\
\hline Residents involved & 23039 & 21781 & 19992 & 20370 & 85182 \\
\hline Mean (range) No of residents per household & $4.3(1-25)$ & $4.2(1-16)$ & $4.9(1-21)$ & $5.0(1-21)$ & $4.5(1-25)$ \\
\hline Children aged 3-9 contributing saliva samples & 2626 & 2230 & 2320 & 2323 & 9499 \\
\hline Households with saliva samples of children & 1803 & 1563 & 1657 & 1675 & 6698 \\
\hline Mean (range) No of children providing samples per household & $0.47(0-5)$ & $0.42(0-5)$ & $0.56(0-7)$ & $0.56(0-7)$ & $0.50(0-7)$ \\
\hline Mean (range) No of children providing samples per cluster & $40(12-94)$ & $35(11-72)$ & $55(38-75)$ & $56(29-73)$ & $45(11-94)$ \\
\hline Vector breeding sites examined & 21988 & 21088 & 13545 & 13767 & 70388 \\
\hline Mean (range) No of breeding sites per household & $0.15(0-10)$ & $0.24(0-10)$ & $0.22(0-24)$ & $0.33(0-24)$ & $0.23(0-24)$ \\
\hline \multicolumn{6}{|l|}{ Missing data: } \\
\hline Households declined to participate & 570 & 596 & 122 & 126 & 1414 \\
\hline Children declined to provide saliva sample & 7 & 20 & 6 & 4 & 37 \\
\hline Children lost to follow-up second sample & 269 & 325 & 142 & 141 & 877 \\
\hline
\end{tabular}


(95\% confidence interval), $\mathrm{P}$ value and degrees of freedom, and intraclass correlation coefficient. Table B in appendix 1 shows the cluster-specific rates used to compute these values. Table $\mathrm{C}$ in appendix 1 provides a mixed effects household level analysis with the intervention as the fixed effect and cluster as random effect.

\section{Secondary outcomes}

In a cluster analysis, residents in intervention clusters were more likely than those in control clusters to believe that communities can control dengue on their own and less likely to believe pesticides are the best way to deal with mosquitoes. Households in intervention clusters were also less likely to buy pesticide (table 3 ).

Per protocol secondary analysis with household as the unit of analysis investigated serological evidence of risk of dengue virus infection in children aged 3-9. Bivariate analysis identified several associations (table 4). In a multivariate analysis of these variables, with country and cluster as random effects, living in an intervention site (adjusted odds ratio 0.74, 95\% confidence interval 0.59 to 0.93 ) and temephos in water containers $(1.44,1.20$ to 1.72$)$ remained in the final model (unadjusted odds ratio 1.49, 1.22 to 1.83; table 4). Temephos in household water containers was associated with higher levels of serological evidence of infection. Unadjusted data show $16.8 \%(238 / 1418)$ of households with temephos and 11.9\% (613/5156) without temephos during the entomological assessment had at least one child with serological evidence of infection. To test if this might be explained by vector control authorities applying temephos disproportionately in households reporting dengue cases, we repeated the analysis of serological evidence in households reporting no known cases in the past year. This produced similar results (adjusted odds ratio 1.44, 1.19 to 1.73). A cluster adjusted household level analysis of control sites showed that, in households where the entomological assessment found temephos in water containers, people were significantly less likely to say they had participated in community activities to control dengue: 501/2222 households with temephos and 1870/6696 households without said they had participated (cluster adjusted odds ratio 0.75, 95\% confidence interval 0.58 to 0.97 ).

\section{Ancillary analysis}

Per protocol subgroup analysis focused on regional differences, especially in rural communities. When we repeated the primary analysis for rural coastal communities (Costa Grande and Costa Chica regions of Guerrero, Mexico) across all intervention communities, 5.4\% (72/1332) of households had a child showing serological evidence of

Table 3 | Cluster analysis for primary and secondary outcomes and intention to treat, with cluster as unit of analysis (risk difference (RD) across clusters, relative risk reduction (RRR), and intraclass correlation coefficient (ICC))*

\begin{tabular}{|c|c|c|c|c|c|c|}
\hline & $\begin{array}{l}\text { Mean in intervention } \\
\text { clusters }(n=75)\end{array}$ & $\begin{array}{l}\text { Mean in control } \\
\text { clusters }(n=75)\end{array}$ & $\mathrm{RD}(95 \% \mathrm{Cl})$ & $\mathrm{RRR}+(95 \% \mathrm{Cl})$ & $\begin{array}{l}\text { P value (df) for } \\
\text { cluster } t \text { test }\end{array}$ & ICC \\
\hline \multicolumn{7}{|l|}{ Primary outcomes } \\
\hline $\begin{array}{l}\text { Serology§: household evidence of recent dengue virus } \\
\text { infection, children aged } 3-9, \geq 2 \times \text { increase of IgG across } \\
\text { paired samples }\end{array}$ & $11.3 \%$ & $14.6 \%$ & $-3.3(-4.9$ to -1.7$)$ & 29.5 (3.8 to 55.3) & $0.038(148)$ & 0.031 \\
\hline $\begin{array}{l}\text { Self reported dengue illness: households reporting in past } \\
\text { year/responding households }\end{array}$ & $5.7 \%$ & $7.1 \%$ & $-1.4(-2.1$ to -0.7$)$ & 24.7 (1.8 to 51.2) & $0.039(148)$ & 0.021 \\
\hline $\begin{array}{l}\text { House index: houses infested with larvae or pupae/ } \\
\text { houses inspected }\end{array}$ & $13.6 \%$ & $19.6 \%$ & $-6.0(-7.1$ to -5.0$)$ & 44.1 (13.6 to 74.7$)$ & 0.001 (148) & 0.075 \\
\hline $\begin{array}{l}\text { Container index: containers with larvae or pupae/ } \\
\text { containers inspected }\end{array}$ & $5.3 \%$ & $8.0 \%$ & $-2.7(-3.9$ to -1.5$)$ & 36.7 (24.5 to 44.8) & $0.001(148)$ & 0.078 \\
\hline $\begin{array}{l}\text { Breteau index: containers with larvae or pupae/houses } \\
\text { inspected }\end{array}$ & $19.7 \%$ & $30.2 \%$ & $-10.5(-17.6$ to -3.4$)$ & 35.1 (16.7 to 55.5) & $0.001(148)$ & 0.061 \\
\hline $\begin{array}{l}\text { Pupae per person index: No of pupae/residential } \\
\text { population } \times 100\end{array}$ & $9.2 \%$ & $17.5 \%$ & $-8.3(-13.4$ to -3.2$)$ & 51.7 (36.2 to 76.1$)$ & $0.001(148)$ & 0.068 \\
\hline \multicolumn{7}{|c|}{ Secondary outcomes (household responses to administered questionnaire) } \\
\hline $\begin{array}{l}\text { Conscious knowledge: recognize sample of larva and } \\
\text { know its relevance (Mexico only) }\end{array}$ & $98.4 \%$ & $97.5 \%$ & 0.9 (0.1 to 1.8$)$ & $1(0.1$ to 1.8$)$ & $0.059(88)$ & 0.097 \\
\hline $\begin{array}{l}\text { Opinion of pesticides: agree (direct question) that } \\
\text { temephos and fumigation are best way to avoid } \\
\text { mosquitoes/households interviewed }\end{array}$ & $80 \%$ & $82 \%$ & $-3.2(-3.8$ to -1.5$)$ & $-3.4(-6.5$ to -0.2$)$ & $0.018(148)$ & 0.029 \\
\hline $\begin{array}{l}\text { Subjective norm: your neighbors believe it worthwhile to } \\
\text { put time and energy into eliminating breeding sites in their } \\
\text { homes (Mexico only) }\end{array}$ & $70.6 \%$ & $68.8 \%$ & $1.8(-0.1$ to 3.8$)$ & $2.6(-4.0$ to 9.1$)$ & $0.43(88)$ & 0.066 \\
\hline $\begin{array}{l}\text { Intention to change: do you plan to dedicate time and } \\
\text { money each week to eliminate breeding sites (Mexico)? }\end{array}$ & $81 \%$ & $78.4 \%$ & $2.6(-1.0$ to 6.3$)$ & $3.2(-1.2$ to 7.7$)$ & $0.158(88)$ & 0.071 \\
\hline $\begin{array}{l}\text { Collective self efficacy: agree communities can themselves } \\
\text { control dengue/households interviewed }\end{array}$ & $48 \%$ & $44 \%$ & 4.7 (3.2 to 6.1) & 9.6 (3.4 to 15.8$)$ & $0.002(148)$ & 0.030 \\
\hline $\begin{array}{l}\text { Socialization/discussion: talk with neighbors about how to } \\
\text { avoid mosquitoes }\end{array}$ & $42 \%$ & $39 \%$ & $3.2(-1.7$ to 81$)$ & $7.5(-3.5$ to 18.6$)$ & $0.341(148)$ & 0.087 \\
\hline $\begin{array}{l}\text { Purchased pesticide: households that purchased in past } \\
\text { month/households interviewed }\end{array}$ & $51 \%$ & $55 \%$ & $-4.0(-5.9$ to -3.0$)$ & $-8.8(-15.4$ to -1.2$)$ & $0.011(148)$ & 0.032 \\
\hline $\begin{array}{l}\text { Social capital: neighbours in this street help one } \\
\text { another out }\end{array}$ & $63.2 \%$ & $62.4 \%$ & $0.9(-0.5$ to 2.2$)$ & $1.3(-4.4$ to 7.1$)$ & $0.51(148)$ & 0.048 \\
\hline
\end{tabular}

*Full cluster specific results for primary outcomes are provided in appendix 1 table B.

tRRR=1-RR.

FICC estimated for control group.

§Proportion of households with positive case, not total positive cases 


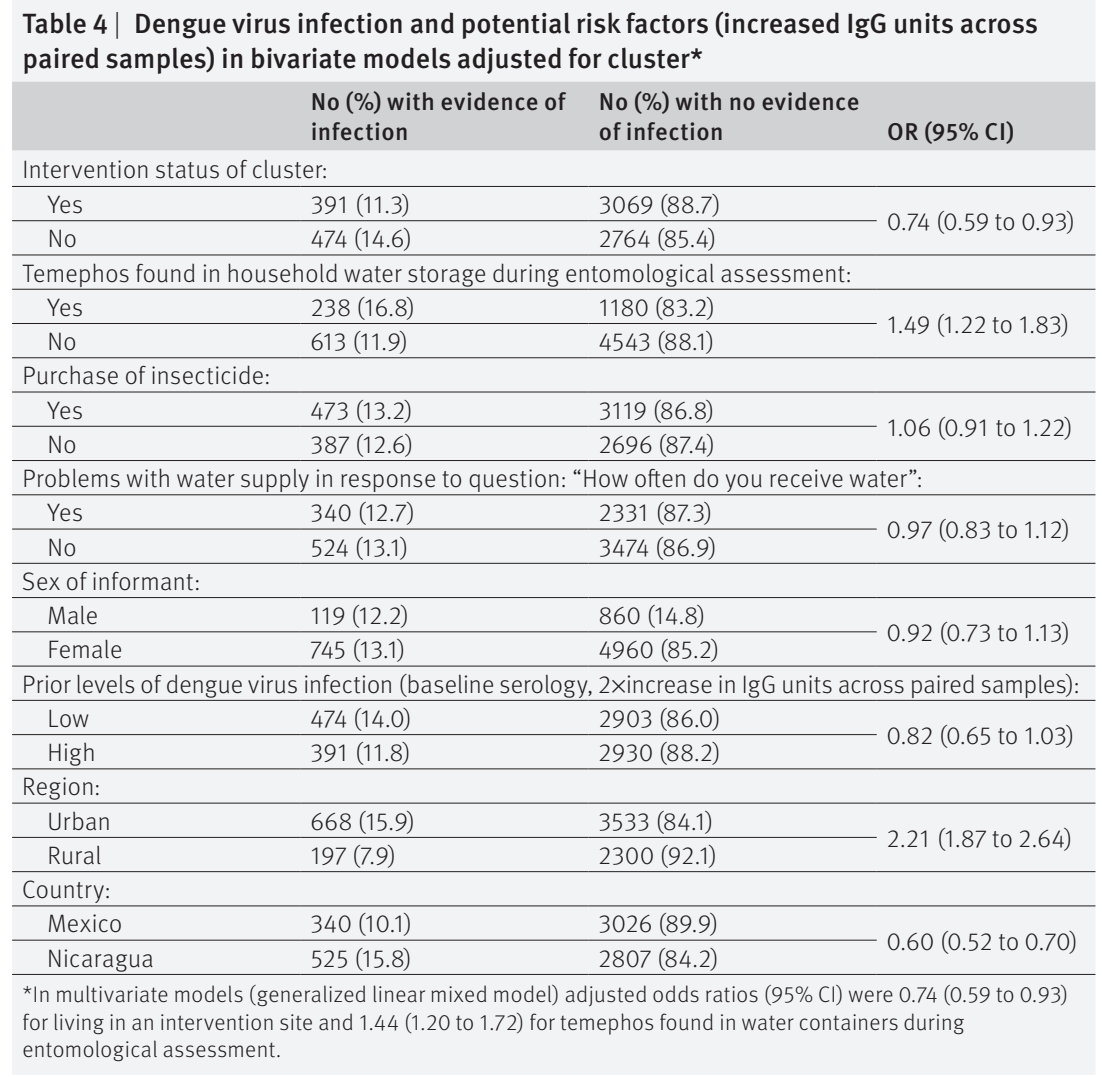

infection compared with $10.7 \%$ (125/1165) in control communities (cluster $t$ test $=-2.949,58 \mathrm{df}, \mathrm{P}=0.005$ ).

Multiple imputation of non-respondents to question about recent dengue cases reconciled 10 datasets (1000 iterations each) to generate almost identical data to those from the original dataset (difference -0.012 , 95\% confidence interval -0.020 to -0.004$)$.

\section{Discussion}

\section{Principal findings}

Informed community mobilization adds effectiveness to government run dengue control. The Camino Verde project reduced $A$ aegypti larvae and pupae and protected against dengue virus infection. To the best of our knowledge, this is the first trial that used serological evidence of recent childhood infection and self reported dengue cases to show an impact of community mobilization on infection with dengue virus.

Our finding that temephos was a risk factor for infection with dengue virus in a prespecified supplementary analysis merits further comment. Reporting bias is unlikely as serological evidence of recent infection was invisible to respondents and health services alike. Consistency of the findings in households reporting no case of dengue in the previous year makes it difficult to explain by vector control programs placing the temephos in response to reported cases. Possibly the increased risk of infection results from a false sense of security engendered by knowledge of pesticide in water storage containers; this could demotivate temephos users from taking physical measures to deal with mosquitoes.

\section{Strengths and limitations of the study}

The serological methods using saliva samples were based on our previous community based study ${ }^{19}$ that compared several serological assays and sample types to determine the best approach to detect incidence of dengue virus infection in paired saliva samples.

A large scale intervention could have a greater impact than we measured, as we returned serology and entomology results to all households before randomization; this probably mobilized intervention and control communities alike. If entomological evaluators knew the intervention status of communities and felt a vested interest, this could bias their assessment. An intensive government anti-dengue campaign in Managua during the impact assessment almost certainly reduced the contrast between intervention and control, as did inclusion of two non-participating clusters assigned to the intervention group. The public security situation in Mexico restricted access to intervention communities and limited community engagement. Exclusion of wealthy Nicaraguan communities precludes conclusions about this social segment.

\section{Comparison with other studies}

Our results are comparable with those in published randomized controlled trials of community participation and entomological indices. ${ }^{101129-32}$

\section{Conclusions and policy implications}

The strong Nicaraguan history of community engagement facilitated success there, but the trial was also successful in Mexico, with quite different community dynamics. In both countries, CIET's strong background in community engagement likely favored success. Nevertheless, the Camino Verde approach might have wider relevance in a range of geographic, cultural, and security settings. We believe the next step is for governments in dengue-endemic countries to implement a similar approach.

We do not, however, expect community participation in dengue control to be easy or easily sustainable. The intervention protocol that engages leadership and community members in discussing evidence and defining local strategies is a promising starting point for a wide range of settings. Each site implementing the intervention in its own way has the advantage of local customization and strong community engagement.

Because of this, a leading question for future research is how best to integrate dengue control within primary healthcare. In contrast with current largely vertical programs distributing temephos or fumigating, this implies dengue control should be rebuilt with fuller community engagement, collaboration with schools, and operational integration with local/municipal services like water supply and garbage disposal.

\section{AUTHOR AFFILIATIONS}

${ }^{1}$ Centro de Investigación de Enfermedades Tropicales (CIET), Universidad Autónoma de Guerrero, Acapulco, Mexico

2Department of Family Medicine, McGill University, 5858 Côte-desNeiges, Montreal, Canada 
${ }^{3} \mathrm{CIET}$, Managua, Nicaragua

4Facultad de Ciencias Biológicas, Universidad Autónoma de Nuevo León, Monterrey, Mexico

${ }^{5}$ Laboratorio Nacional de Virología, Centro Nacional de Diagnóstico y Referencia, Ministerio de Salud, Managua, Nicaragua

${ }^{6}$ Departamento de Prevención y Control de Enfermedades Transmisibles por Vector, Servicios Estatales de Salud Guerrero, Av Rufo Figueroa 6, Colonia Burócratas, Chilpancingo, Mexico. ${ }^{7}$ Division of Infectious Diseases and Vaccinology, School of Public Health, University of California, Berkeley, Berkeley, CA, USA ${ }^{8}$ CIET International, New York, NY, USA

Contributors: NA was principal investigator in the Mexican trial, wrote the proposals, contributed to design and coordination, did the analysis, and prepared this article. ENA was lead researcher in Mexico and contributed to the design and drafting. JA was lead researcher in the Nicaragua feasibility study and the trial and contributed to the trial design and analysis and drafting of this article. AMP coordinated fieldwork in Mexico and contributed to the design and the drafting of this article. HS coordinated fieldwork in Nicaragua and contributed to the design and the drafting of this article. JLS, AJCG, RSS, SP, CH, IFS, and $A B$ contributed to the fieldwork, analysis, and drafting. JC contributed to the proposals and drafting this article and was a co-investigator in the Nicaragua feasibility study. RJL contributed to the trial design, provided coordination between Nicaragua and Mexican teams, and contributed to the design and drafting of this paper. EH was principal investigator in the Nicaraguan feasibility study and trial and contributed to the proposals and drafting of this article. NA is guarantor.

Funding: The UBS Optimus Foundation provided funding for the feasibility study in Nicaragua and the full trial in both Nicaragua and Mexico. Fomix-CONACYT-GUERRERO supported the work in Acapulco (grant No 2008-02-108541). The sponsors had no role in study design, in collection or interpretation of data, in writing the report, or in the decision to submit the article for publication.

Competing interests: All authors have completed the ICMJE uniform disclosure form at www.icmje.org/coi disclosure.pdf and declare: no support from any organization for the submitted work; no financial relationships with any organizations that might have an interest in the submitted work in the previous three years; no other relationships or activities that could appear to have influenced the submitted work.

Ethical approval: This study was approved by institutional review boards at the University of California, Berkeley (22 July 2010), the Nicaraguan Ministry of Health (25 August 2010), and CIETinternational (1 August 2010) for the Nicaraguan study and the CIETcanada research ethics board (16 November 2009) and the ethics committee of the Centro de Investigación de Enfermedades Tropicales at the Universidad Autónoma de Guerrero (27 November 2009) for the Mexican study. All boards performed annual review and approval throughout the study. All participants gave informed consent.

Transparency declaration: NA (the manuscript's guarantor) affirms that the manuscript is an honest, accurate, and transparent account of the study being reported; that no important aspects of the study have been omitted; and that any discrepancies from the study as planned have been explained.

Data sharing: No additional data available.

This is an Open Access article distributed in accordance with the Creative Commons Attribution Non Commercial (CC BY-NC 4.0) license, which permits others to distribute, remix, adapt, build upon this work non-commercially, and license their derivative works on different terms, provided the original work is properly cited and the use is noncommercial. See: http://creativecommons.org/licenses/by-nc/4.0/.

1 Brady OJ, Gething PW, Bhatt S, et al. Refining the global spatial limits of dengue virus transmission by evidence-based consensus. PLoS Negl Trop Dis 2012:6:e1760.

2 Bhatt S, Gething PW, Brady OJ, et al. The global distribution and burden of dengue. Nature 2013;496:504-7.

3 WHO. Dengue: guidelines for diagnosis, treatment, prevention and control. World Health Organization, 2009.

4 Esu E, Lenhart A, Smith L, et al. Effectiveness of peridomestic space spraying with insecticide on dengue transmission; systematic review. Trop Med Int Health 2010:15:619-31.

5 Braga IA, Lima JBP, da Silva Soares S, et al. Aedes aegypti resistance to temephos during 2001 in several municipalities in the states of Rio de Janeiro, Sergipe, and Alagoas, Brazil. Mem Inst Oswaldo Cruz 2004;99:199-203.

6 Jirakanjanakit B, Saengtharatip S, Rongnoparut P, et al. Trend of Temephos resistance in Aedes aegypti mosquitoes in Thailand during 2003-2005. Environ Entomol 2007:36:506-11.
7 Lima JBP, da Cunha MP, da Silva Jr RC, et al. Resistance of Aedes aegypti to organophosphates in several municipalities in the state of Rio de Janeiro and Espirito Santo, Brazil. Am I Trop Med Hyg 2003:68:329-33.

8 Rodriguez MM, Bisset JA, Fernandez D. Levels of insecticide resistance and resistance mechanisms in Aedes aegypti from some Latin American countries. J Am Mosa Control Assoc 2007:23:420-9.

9 Heintzea C, Velasco Garridob M, Kroeger A. What do communitybased dengue control programmes achieve? A systematic review of published evaluations. Trans R Soc Trop Med Hyg 2007;101:317-25.

10 Vanlerberghe V, Toledo ME, Rodríguez M, et al. Community involvement in dengue vector control: cluster randomised trial. BMJ 2009;338:1959.

11 Arunachalam N, Tyagi BK, Samuel M, et al. Community-based control of Aedes aegypti by adoption of eco-health methods in Chennai City, India. Pathog Glob Health 2012;106:488-96.

12 Kittayapong P, Thongyuan S, Olanratmanee P, et al. Application of eco-friendly tools and eco-bio-social strategies to control dengue vectors in urban and peri-urban settings in Thailand. Pathog Glob Health 2012;106:446-54

13 Al-Muhandis N, Hunter PR. The value of educational messages embedded in a community-based approach to combat dengue fever: a systematic review and meta-regression analysis. PLoS Negl Trop Dis 2011;5:e1278.

14 Boyce R, Lenhart A, Kroeger A, et al. Bacillus thuringiensis israelensis(Bti) for the control of dengue vectors: systematic literature review. Trop Med Int Health 2013:18:564-77

15 Arostegui J, Suazo H, Coloma J, et al. Impact on serological, entomological, and behavioral indices of an evidence-based community-derived communication programme for the control of Aedes aegypti and dengue in Managua, Nicaragua. Am I Trop Med Hyg 2008;79(6 suppl):216-7.

16 Califf RM, Woodlief LH. Pragmatic and mechanistic trials. Eur Heart J 1997;9:367-70

17 Daniel J. Sampling essentials. Practical guidelines for making sampling choices. Howard University/Sage 2012:140. www.sagepub. com/upm-data/40803 5.pdf.

18 Hawe P, Shiell A, Riley T. Complex interventions: how "out of control" can a randomised controlled trial be? BMJ 2004;328:1561-3.

19 Balmaseda A, Saborio S, Tellez Y, et al. Evaluation of serological markers in serum, filter-paper bloodspots, and saliva for dengue diagnosis and epidemiological studies. Clin Virol 2008:43:287-91.

20 Kuan G, Gordon AL, Aviles W, et al. The Nicaraguan Pediatric Dengue Cohort Study: Study design, methods, use of information technology, and extension to other infectious diseases. Am J Epidemiol 2009;170:120-9.

21 Balmaseda A, Mercado JC, Matute JC, et al. Trends in patterns of dengue transmission in a pediatric cohort study in Nicaragua. I Infect Dis 2010;201:5-14

22 Taylor DW, Bosch EG. CTS: a clinical trials simulator. Stat Med 1990;9:787-801.

23 Campbell MK, Mollison J, Steen N, et al. Analysis of cluster randomized trials in primary care: a practical approach. Fam Pract 2000;17:192-6.

24 Donner A. Klar N. Confidence interval construction for effect measures arising from cluster randomization trials. / Clin Epidemiol 1993:46:123-31

25 Honaker J, King G, Blackwell M. Amelia II: a program for missing data. http://gking.harvard.edu/amelia.

26 Rubin DB. Multiple imputation for non-response in surveys. John Wiley, 1987.

27 Imai K, King G, Lau O. Zelig: everyone's statistical software, 2007. http://gking.harvard.edu/zelig.

28 Andersson N, Mitchell S. Epidemiological geomatics in evaluation of mine risk education in Afghanistan: introducing population weighted raster maps. Int J Health Geogr 2006;5:1.

29 Espinoza-Gomez F, Hernandez-Suarez CM, Coll-Cardenas R. Educational campaign versus malathion spraying for the control of Aedes aegypti in Colima, Mexico. J Epidemiol Community Health 2002;56:148-52.

30 Lloyd LS, Winch P, Ortega-Canto J, et al. Results of a community-based Aedes aegypti control program in Merida, Yucatan, Mexico. Am / Trop Med Hyg 1992;46:635-42

31 Leontsini E, Gil E, Kendall C, Clark GG. Effect of a community-based Aedes aegypti control programme on mosquito larval production sites in El Progreso, Honduras. Trans Roval Soc Trop Med Hyg 1993;87:267-71.

32 Castro M, Sanchez L, Perez D, et al. A community empowerment strategy embedded in a routine dengue vector control programme: a cluster randomised controlled trial. Trans R Soc Trop Med Hyg 2012:106:315-21

(c) BMJ Publishing Group Ltd 2015

Appendix 1: Supplementary tables A-C

Appendix 2: Details of the Camino Verde intervention

Appendix 3: Primary outcomes and confidence intervals for each group with cluster as unit of analysis 